J. W. R. Pott

J. Van Hof-van Duin

D. J. Heersema

W. P. F. Fetter

A. M. Schreuder

S. P. Verloove-Vanhorick

Received: 28 March 1994

Accepted: 27 September 1994

J. W. R. Pott - J. Van Hof-van Duin (区)

D. J. Heersema

Department of Physiology I,

Erasmus University Rotterdam,

PO Box 1738,

NL-3000 DR Rotterdam,

The Netherlands

Fax: 31-10-4367594

W. P. F. Fetter

Neonatal Unit, Sophia Hospital, Zwolle, The Netherlands

\section{A. M. Schreuder}

Department of Paediatrics,

Division of Neonatology,

Sophia Children's Hospital

and Erasmus University Rotterdam,

The Netherlands

S. P. Verloove-Vanhorick

Department of Child Health,

TNO Institute of Preventive Health Care,

Leiden, The Netherlands

\title{
Strabismus in very low birth weight and/or very preterm children: discrepancy between age of onset and start of treatment
}

Abstract Very low birth weight (VLBW; less than $1500 \mathrm{~g}$ ) and /or very preterm children are at risk for strabismus. However, the age of onset of strabismus is still unknown. The present study reports on the frequency of strabismus in 4505 -yearold children born with a birth weight of less then $1500 \mathrm{~g}$ and/or with a gestational age below 32 weeks. The age at which strabismus was initially diagnosed was determined retrospectively. At 5 years of age 65 of the atrisk children $(14.4 \%)$ presented with strabismus. Fifteen of them (3\%) had at that age not been referred to or treated by an ophthalmologist. At 3 years of age only 28 strabismic children were being treated; 7 at-risk children $(2 \%)$ had been treated for strabismus before the end of the 1 st year. These results were compared to those from a second study in which eye alignment was longitudinally examined in 194 VLBW children from 6 weeks until 12 months of (corrected) age and additionally in 65 of these children at the age of 2.5 years. At a first glance, the frequency of strabismus in the longitudinal study seemed rather stable during the first 2.5 years of life, with values varying between $14 \%$ and $18 \%$. However, only a small percentage of misalignments which were noted at 6 weeks of age persisted until 2.5 years. On the other hand, if children had a misalignment at 9 months, strabismus was still present when they were reexamined at 1 and at 2.5 years of age. In order to prevent the rather high incidence of untreated strabismus, screening programmes for atrisk children should focus on diagnosing strabismus at 9 months of (corrected) age. Because strabismus can also develop after this age, it is important to re-examine ocular alignment at later ages.

Conctirsion mines nedical oale is

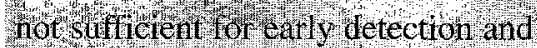
treativent of strabismus in at $-r i s k$ chilen. Our results suggest that the optinal screening age for early deterion of persistent sirabismus in VLI W children is at 9 nionths of age, Because strabismus can also derelop after ths ase, It is 1 mportan Horept exanminon of visual runctons of at wis-chilen at reglilar intervals after 1 year of age.

Key words Very low birth weight Strabismus · Vision screening

Abbreviations $O K N$ optokinetic nystagmus - POPS Project on preterm and small for gestational age infants in The Netherlands 1983 $V L B W$ very low birth weight (birth weight $<1500 \mathrm{~g}$ ) 
Fig. 1 Prevalence of strabismus in VLBW and control children as reported by various studies. The horizontal axis represents the age at which children were tested, the vertical axis the prevalence of strabismus. Each study is represented by a different symbol; results obtained in healthy fullterm children are indicated by open symbols and in VLBW children by closed symbols

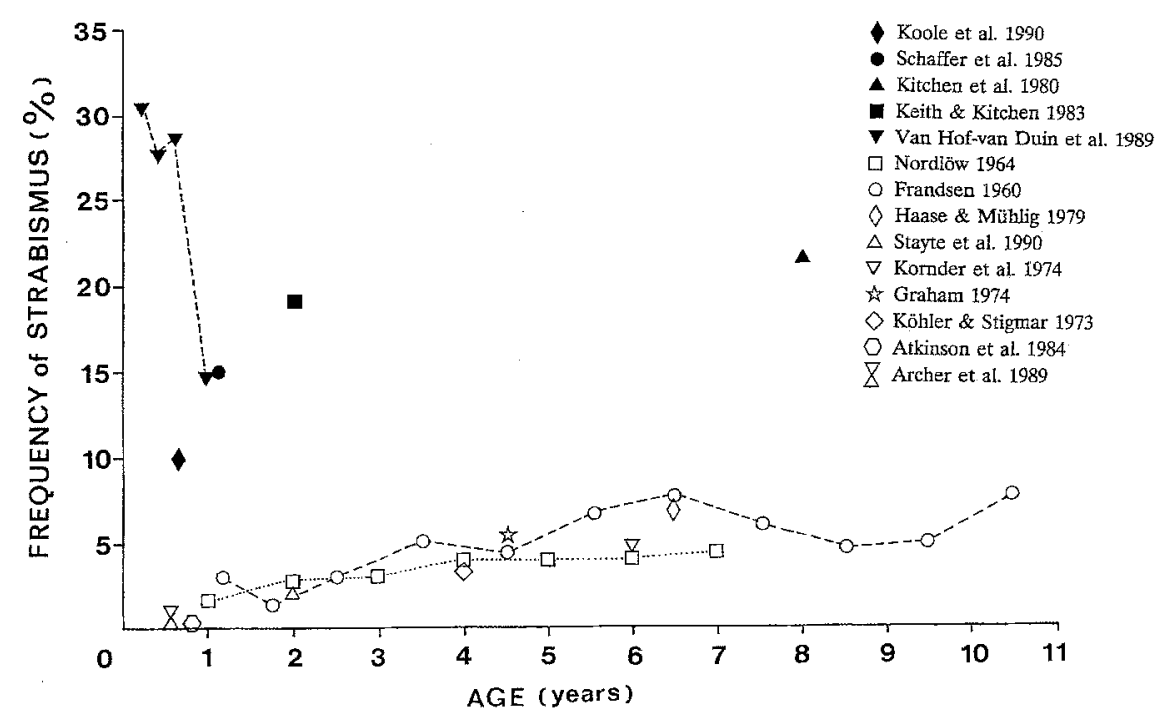

\section{Introduction}

Very low birth weight (VLBW, less than $1500 \mathrm{~g}$ ) and/or very preterm (i.e. after a gestational age below 32 weeks) children are known to be at risk for developing strabismus $[4,7,11,12,14,18,21]$. However, the age of onset of strabismus in these at-risk children is still unknown. Strabismus may cause impaired binocular visual functions and amblyopia. Although the possibilities and methods to restore binocular functions in children with early onset strabismus is still controversial, treatment of amblyopia can be effective when started at an early age. As a consequence, knowledge about the onset of strabismus in atrisk children is essential.

Literature data on the prevalence of strabismus in normal full-term children and in children whose birth weight had been less than $1500 \mathrm{~g}$ (VLBW) are illustrated in Fig. 1. Archer et al. [1] reported that in normal full-term infants early onset strabismus becomes evident between the 2nd and 4th month of life, and that the prevalence of permanent strabismus is $0.5 \%$ at 6 months of age. Other authors showed a gradual increase of strabismus in full-term children with values of $3 \%-5 \%$ at 5 years of age $[2,5,6$, $8,9,13,15,17,20]$.

In VLBW children the prevalence of strabismus is much higher. In a retrospective, mainly cross-sectional study on the effect of VLBW on visual development during the 1st year after term, Van Hof-van Duin et al. [21] reported a high incidence of strabismus convergens in VLBW infants between 3 and 9 months of corrected age (28\%$30 \%$ ), followed by a decrease to $14.5 \%$ at 1 year. As shown in Fig. 1, most authors reported $10 \%-20 \%$ strabismus in VLBW children of 9 months up to 8 years of age $[4,7,11,12,14,18]$. Until now longitudinal studies on the frequency of strabismus in VLBW children during the first years of life are lacking.
The present cross-sectional study reports on the frequency of strabismus in 5-years-old at-risk children who were born with a birth weight of less then $1500 \mathrm{~g}$ and/or after a gestational period below 32 weeks. The age at which strabismus was first diagnosed was determined retrospectively. We also present results from a second study in which the incidence of strabismus in VLBW children was followed longitudinally from 6 weeks until 12 months of life, and in some of them until the age of 2.5 years. Comparison of the results from both studies will show whether discrepancies exist between age of onset of strabismus and start of treatment; as a consequence they will give an indication whether or not the present care for atrisk children is sufficient for early detection of strabismus.

\section{Patients and methods}

The first study population consisted of a geographical subpopulation of a nationwide prospective study, the Project on preterm and small for gestational age infants (POPS), concerning all infants $(n=$ 1,338 , born alive in The Netherlands in 1983 with a birth weight of less than $1500 \mathrm{~g}$ (i.e. VLBW) and/or after a gestational age below 32 completed weeks $[3,22]$. Of this national study, 541 children, who lived within reasonable travelling distance from Rotterdam but were otherwise representative of the entire population of 966 surviving 5-year-olds, were invited to co-operate with a cross-sectional study on the long-term effects of VLBW and/or very preterm birth on visual development. An extensive visual examination, including the assessment of eye alignment, was carried out in 450 out of the 541 invited children $(83 \%)$ at the department of Physiology I of the Erasmus University Rotterdam. Although parents of 91 children chose not to participate, the reasons of their refusal did not seem to bias the outcome. Birth weights of the 450 at-risk children ranged from 560 to $2580 \mathrm{~g}$ (median $1335 \mathrm{~g}$ ) and gestational ages ranged from 26 to 38.8 weeks (median 31 weeks); 364 children were born with a VLBW, 303 children after a gestational period below 32 weeks and 217 were both VLBW and very premature.

The second, longitudinal study consisted of 194 out of all (278) VLBW children who were admitted to the neonatal intensive care unit of the Sophia Children's Hospital in Rotterdam between 1 Au- 
gust 1985 and 1 January 1988. This unit serves as a tertiary referral centre for the South West of the Netherlands with approximately 25000 deliveries per year. Visual functions (including the presence or absence of strabismic deviations) were tested longitudinally in 194 of the 222 surviving infants at 6 weeks, 3, 6, 9, and 12 months of corrected age during out-patient visits to the hospital for paediatric follow up. Additionally, a subgroup of 65 out of the 81 VLBW children, who were born between 1 August 1985 and 1 August 1986 and survived the 1st year of life, were visually assessed at 2.5 years of life. Birth weights of the 194 VLBW children ranged from 660 to $1499 \mathrm{~g}$ (median $1160 \mathrm{~g}$ ) and gestational ages from 24.8 to 37 weeks (median 30.3 weeks).

In both study groups the presence of strabismus was assessed by examining symmetry of the corneal light reflex (Hirschberg's method) and by using the cover test [23].

\section{Results}

At 5 years of age 65 out of the 450 at-risk children of the first, cross-sectional study $(14.4 \%)$ presented with strabismus; 46 children $(10.2 \%)$ were convergent (of whom 40 continuous and 6 intermittent) and $19(4.2 \%)$ divergent (5 continuous and 14 intermittent). The ratio convergent/divergent strabismus was 2.4 . In 2 additional children the eyes were found aligned at the age of 5 years, after surgical correction for esotropia at earlier ages. The distribution of strabismus amongst the various subgroups was not statistically different $\left(\chi^{2}>0,05\right)$, with a frequency of strabismus of $14.8 \%$ in VLBW children $(n=364), 14.9 \%$ in children born after a gestational period below 32 weeks $(n=303)$, and $15.7 \%$ in children who were both very preterm and VLBW $(n=217)$.

In order to determine the age at which strabismus had been diagnosed for the very first time, information was obtained with the parents' consent, from all ophthalmologists to whom the children had been referred. Fifty-two out of the 65 children with strabismus at 5 years had been or were treated by an ophthalmologist. Ophthalmological information was received on 46 children. In the remaining 6 children information obtained from the parents could be used to establish a reasonable estimation of the age strabismus had appeared. Seven children of the 65 5-yearolds with strabismus $(10.8 \%)$ were seen by an ophthalmologist for strabismus during the 1st year of life; 28 at 3 years of age $(43.1 \%), 37$ at 4 years $(56.9 \%)$ and 50 at 5 years $(76.9 \%)$. However, at 5 years 15 of the 65 strabismic children (23\%) had never been seen by an ophthalmologist. Nine of these children showed intermittent and six continuous strabismus.

In the second study 194 VLBW children were tested longitudinally during the first 12 months after term (17 were tested once, 17 twice, 18 three times, 73 four times and 69 at all five test ages). The percentages of children actually tested for strabismus ranged between $68 \%$ at 9 months and $79 \%$ at 6 months (see Table 1 ). At 2.5 years of (corrected) age 65 of the intended 81 children (80\%) were tested. As shown in Table 1, the frequency of strabismus was rather stable during the first 2.5 years of age, with
Table 1 Numbers of tested children and prevalence of strabismus in 194 VLBW children tested longitudinally during the first 12 months of life (corrected age) and in a subgroup of these children at 2.5 years

\begin{tabular}{|c|c|c|c|c|}
\hline & $\begin{array}{l}\text { Children } \\
\text { tested }\end{array}$ & $\begin{array}{l}\text { Convergent } \\
\text { strabismus }\end{array}$ & $\begin{array}{l}\text { Divergent } \\
\text { strabismus }\end{array}$ & $\begin{array}{l}\text { Total } \\
\text { strabismus }\end{array}$ \\
\hline 6 weeks & $145(74.7 \%)$ & $10(6.9 \%)$ & $16(11.0 \%)$ & $26(17.9 \%)$ \\
\hline 3 months & $147(75.7 \%$ & $18(11.2 \%)$ & $7(4.8 \%)$ & $25(17.0 \%)$ \\
\hline 6 months & $153(78.9 \%)$ & $15(9.8 \%)$ & $8 \quad(5.2 \%)$ & $23(15.0 \%)$ \\
\hline 9 months & $132(68.0 \%)$ & 13. $(9.8 \%)$ & $6(4.6 \%)$ & $19(14.4 \%)$ \\
\hline 1 year & $144(74.2 \%)$ & $18(12.5 \%)$ & $4(2.8 \%)$ & $22(15.3 \%)$ \\
\hline 2.5 years & $65(80.3 \%)$ & $9(13.8 \%)$ & $2(3.0 \%)$ & $11(16.9 \%)$ \\
\hline
\end{tabular}

Prevalence of strabismus in 450 VLBW and/or very preterm born children tested cross-sectionally at 5 years of age

$\begin{array}{llllll}5 \text { years } & 450 & 46(10.2 \%) & 19 & (4.2 \%) & 65(14.4 \%)\end{array}$

a Excluding 2 cases who had been surgically corrected at earlier ages

values varying between $14 \%$ and $18 \%$. The lowest prevalence occurred at 9 months.

A remarkable finding was, however, that some of the children with either divergent or convergent strabismus at 6 weeks of (corrected) age were found aligned at later ages. On the other hand, in other children strabismus was not present at 6 weeks and/or 3 months, but developed at a later age. In order to further evaluate this phenomenon the prevalence and incidence of strabismus were compared at the different testing ages.

At 6 weeks, 26 out of 145 (17.9\%) tested children had strabismus. However, in only 5 did strabismus remain present at all later test ages; in 1 child strabismus was still present at 3 months but had disappeared at later test ages and in 13 children strabismus had disappeared from 3 months onward; in 4 cases strabismus had resolved at 3 months, but reappeared later on. Unfortunately, the course of strabismus could not be evaluated in 3 children because of lack of follow up. Of the 119 children with a normal eye-alignment at 6 weeks, 19 presented with strabismus at a later age. Furthermore, of the 49 children who were not tested at 6 weeks, at least 13 had strabismus at one or more examinations later on.

At 3 months, 25 out of $147(17 \%)$ tested children had strabismus, of whom in 19 cases strabismus was diagnosed for the first time. Strabismus remained present at later examinations in 14 of the 25 children. On the other hand, strabismus disappeared later in 9 children (in 8 cases from 6 months onward and in one child after 6 months). Follow up was lacking in 2 cases. In 11 of 122 children with normal eye alignment at 3 months, strabismus developed at a later age. Of the 47 children who were not tested at 3 months, at least 8 presented with strabismus at later test ages.

At 6 months, 23 of the 153 tested children (15.0\%) were strabismic, of whom in 8 children strabismus was 
noted for the first time. Strabismus remained present at all later examinations in 16 of the 23 cases, but had disappeared in 3 of them at 9 months, whereas no follow up was possible in 4 children. In 6 of the 130 children with a normal eye-alignment at 6 months strabismus developed at a later age. At least 4 of the 41 children who were not examined at 6 months, had strabismus later on.

At 9 months, 19 of the 132 examined children (14.4\%) had strabismus, of whom in 3 cases strabismus was noted for the first time. In 18 of the 19 cases strabismus was still present at 12 months, whereas follow up was lacking in one strabismic child. In 2 of the 113 children with normal eye-alignment at 9 months, strabismus developed later on. At least 3 of the 62 children who were not tested at 9 months, had strabismus at a later age.

At 12 months, 22 of the 144 tested children (15.3\%) had strabismus, of whom in 2 strabismus was noted for the first time.

At 2.5 years only 65 of the 81 surviving VLBW children born between 1 August 1985 and 1 August 1986 were examined. Strabismus was diagnosed in 11 children (16.9\%), of whom in 2 for the first time, whereas in 9 cases strabismus had been present from 9 months $(n=6)$ and/or 12 months $(n=7)$ onward. One of the 54 children with normal eye-alignment at 2.5 years had been convergent at 12 months.

\section{Discussion}

Our results confirm earlier reports that strabismus is a frequent finding in at-risk children, occurring as often in 5-year-olds born with a VLBW (14.8\%) as in those whose gestational age had been below 32 weeks $(14.9 \%)$. In the longitudinal study of VLBW children, the prevalence of strabismus ranged between $14 \%$ and $18 \%$ from 6 weeks until 2.5 years of age. In an earlier study Van Hofvan Duin et al. [21] reported higher percentages of convergent strabismus in VLBW infants between 3 and 9 months (28\%-30\%) decreasing to $14.5 \%$ at 1 year (see also Fig. 1). However, those results are not comparable to the present ones, becauses they represented a retrospective, mainly cross-sectional study with a bias to re-examine visually impaired children. Furthermore, since in that study only $11 \%$ of the VLBW infants were examined at all test ages, the high percentage of strabismus between 3 and 9 months could be caused, according to the authors, by a spurious statistic due to different infants tested at different ages.

In the present cross-sectional study of 5-year-olds born with a VLBW and/or very preterm, the frequency of strabismus was $14.4 \%$. This frequency is slightly lower than the $19 \%$, recently reported in the nationwide POPS study [3], of which our 450 at-risk children were a geographical subpopulation. This difference can partly be explained by the fact that in the nationwide study patients were exam- ined during home visits by a paediatrician, which allowed examination of non-ambulant children. Secondly, it is possible that during home visits heterophoria was diagnosed as strabismus. In our study a rather high frequency of heterophoria was found to be present in the at-risk children $(41.1 \%)$, whereas the percentage heterophoria in the nationwide study is not mentioned.

Althought the cause of strabismus in VLBW and very preterm born children is still unknown, strabismus seems to be rather of cerebral than of ophthalmological origin. It is generally known that VLBW and/or very preterm children are at risk for pre- and perinatal cerebral disturbances, and an association between cystic periventricular leucomalacia diagnosed by ultrasound and the occurrence of strabismus has been demonstrated [7]. Furthermore, it is known that $40 \%-60 \%$ of children with cerebral palsy will develop strabismus [10].

From the results of the present longitudinal study it is clear that the incidence of strabismus in VLBW children is already very high throughout the 1 st year of life. However, in only 5 out of 26 children with an abnormal eye alignment at 6 weeks of corrected age, strabismus remained present at all later test ages. In addition, as shown in Table 1, eye deviations at 6 weeks were usually divergent. It seems therefore, that misalignments at 6 weeks of age in at-risk children possibly represent a delayed development of conjugated eye movements and binocular fixation. Results of the present study show that most children with unconjungated eye movements at 6 weeks appeared to develop normal eye alignment before $6-9$ months of age. With increasing age the percentage of children with persisting strabismus became higher: at 3 months 14 children $(9.5 \%)$ had permanent strabismus, at 6 months 16 children (10.4\%), and at 9 months 18 children (13.6\%). If misalignments were present at 9 months, they persisted until the age of 2.5 years. On the other hand, the incidence (new cases) of strabismus decreased after 6 weeks of age from 19 children (12.9\%) at 3 months to 2 children (1.4\%) at 1 year, but at 2.5 years, at least 2 other new cases of strabismus $(3.0 \%)$ were diagnosed.

As a consequence, the present results indicate that the optimal age for early detection of persistent strabismus in at-risk children is at 9 months of (corrected) age. However, children should be re-examined at later ages, because new cases of strabismus have been demonstrated in older children. Screening before 3 months of age seems to be inefficient, because many VLBW children tend to have unconjugated eye movements during this period.

Although the results of the present study indicate that strabismus in at-risk children has an early onset (before 6-9 months of corrected age), the characteristics of this strabismus have little resemblance with the so called 'early onset' or 'essential infantile strabismus' as described in full-term children. Characteristics of 'essential infantile strabismus' are amongst others an onset of strabismus before the age of 6 months, dissociated vertical 
deviation, presence of latent nystagmus, cross-fixation and a directional asymmetry of the monocular optokinetic nystagmus $[16,19,24]$. Of the 655 -year-old at-risk children with strabismus in the present study, only 5 children $(1.1 \%)$ showed dissociated vertical deviation, 2 children $(0.4 \%)$ latent nystagmus, 2 children $(0.4 \%)$ cross-fixation, and 34 children $(7.6 \%)$ asymmetrical monocular optokinetic nystagmus.

It is generally accepted that early detection of strabismus is essential for optimal results of treatment and for the prevention of amblyopia. The primary goal in the management of strabismus is to correct associated refractive errors and amblyopia. Secondarily, surgery of eye muscles can be performed to correct larger angles of strabismus. In other words, 'treatment' of strabismus generally includes a period of occlusion therapy, until fixation preference is abolished, followed by surgery. Only rarely is normal eye alignment with normal binocular vision obtained and often a small angle of strabismus will remain. In the present study all 5-year-old at-risk children were routinely followed up by paediatricians from birth until 2 years of (corrected) age; after this age they were included in the Dutch Child Health Care Programme. Despite this health care, most children with strabismus were referred to an ophthalmologist for the first time after the age of 1 year, whereas at 5 years of age, 15 out of 65 strabismic atrisk children had never visited an ophthalmologist. The discrepancy between the time of onset of strabismus and the age of diagnosis might possibly be caused by difficulties in orthoptic examination of small infants. Also, small angle strabismus could remain unnoticed during regular Child Health Care follow up. Moreover, at-risk children with visual dysfunctions often have additional impairments in other sensory or neuromotor functions and mental development, which may delay the first visit to an ophthalmologist. In practice the often long waiting-lists of ophthalmologists may bring about a further delay.

Acknowledgements We would like to thank C. Reus-van Haeren for her administrative and logistic help and her contribution in examining the children, C. W. Eveleens-Maarse and J. P. de Man for the orthoptic examination of the children, ir. W. C. J. Hop for statistical analysis and B. L. F. Weijer for technical assistance. J. W. R. Pott was supported by the Praeventiefonds, 's-Gravenhage, The Netherlands \#28-1544.

\section{References}

1. Archer SM, Sondhi N, Helveston EM (1989) Strabismus in infancy. Ophthalmology 96: 133-137

2. Atkinson J, Braddick OJ, Durden K, Watson PG, Atkinson S (1984) Screening for refractive errors in 6-9 months old infants by photorefraction. $\mathrm{Br} \mathrm{J}$ Ophthalmol 68: 105-112

3. Ens-Dokkum MH, Schreuder AM, Veen S (1993) Outcome at five years of age in very preterm and very low birthweight infants in the Netherlands. Rijksuniversiteit Leiden, Pasmans offsetdrukkerij b.v., The Netherlands

4. Fledelius $H$ (1976) Prematurity and the eye. Acta Ophthalmol 128 [Suppl]: $1-208$

5. Frandsen AD (1960) Occurence of squint. Acta Ophthalmol 62 [Suppl]: $1-149$

6. Friedman Z, Neuman E, Hyams SW, Peleg B (1980) Ophthalmic sreening of 38,000 children, age 1 to $2 \frac{1}{2}$ years, in child welfare clinics. J Pediatr Ophthalmol Strab 75:527-531

7. Gibson NA, Fielder AR, Trounce JQ, Levence MI (1990) Ophthalmic findings in infants of very low birthweight. Dev Med Child Neurol 32:7-13

8. Graham PA (1974) Epidemiology of strabismus. Br J Ophthalmol 58: 224-231

9. Haase W, Mühlig HP (1979) Schielhäufigkeit bei Hamburger Schulanfänger. Klin Monatsbl Augenheilkd $174: 232-235$
10. Harcourt B (1974) Strabismus affecting children with multiple handicaps. Br J Ophthalmol 58:272-280

11. Keith CG, Kitchen WH (1983) Ocular morbidity in infants of very low birthweight. Br J Ophthalmol 67:302-305

12. Kitchen WH, Ryan MM, Richards A, Mcdougal AB, Billson FA, Keir EH (1980) A longitudinal study of very low birthweight infants: an overview of performance at eight years of age. Dev Med Child Neurol 22:172-188

13. Köhler L, Stigmar G (1973) Vision screening of four-years-old-children. Acta Paediatr Scand 62:17-27

14. Koole FD, Bax PP, Samson JF, Van der Lei J (1990) Ocular eamination in nine-month-old infants with very low birthweights. Ophthalmic Paediatr Genet 11:89-94

15. Kornder LD, Nursey JN, Pratt-Johnson JA, Beattie A (1974) Detection of manifest strabismus in young children. Am J Ophthalmol 77:211-214

16. Mein J, Harcourt B (1986) Diagnosis and management of ocular motility. Blackwell Scientific, Oxford

17. Nordlöw W (1964) Squint-the frequency of onset at different ages, and the incidence of some associated defects in a Swedish population. Acta Ophthalmology 42:1015-1037

18. Schaffer DB, Johnson L, Quinn GE, Weston M, Bowen FW (1985) Vitamin $\mathrm{E}$ and retinopathy of prematurity. Ophthalmology 92: 1005-1011
19. Spielman A (1990) Pathophysiology of the symptoms of infantile strabismus. In: Campos EC (ed) Strabismus and ocular motility disorders. McMillan Press, London, pp 209-214

20. Stayte M, Johnson A, Wortham C (1990) Ocular and visual defects in a geographical defined population of 2-years-old children. Br J Ophthalmol $74: 465-468$

21. Van Hof-van Duin J, Evenhuis-van Leunen A, Mohn G, Baerts W, Fetter WPF (1989) Effects of very low birthweight on visual development during the first year after term. Early Hum Dev 20:255-266

22. Veen S, Ens-Dokkum MH, Schreuder AM, Verloove-Vanhorick SP, Brand R, Ruys JH (1991) Impairments, disabilities, and handicaps of very preterm and very-low-birthweight infants at five years of age. Lancet $338: 33-36$

23. Von Noorden GK (1977) Von Noorden-Maumenee's atlas of strabismus, 3rd edn. CV Mosby Co, St. Louis

24. Von Noorden GK (1988) A reassessment of infantile esotropia. XLIV Edward Jackson Memorial Lecture. Am J Ophthalmol 105 : 1-10 